healthy action. Lymphatic inflammation was followed by numerous abscesses about the ellow, which were treated by poultices and incisions, with tonic constitutional remedies. He was discharged well in six wceks.

Case XII.-An infant, 11 weeks old. Polypus nasi removed. This is an interesting case, from the extreme youth of the patient, the polypus having probably commeneed its growth in utero.

Cases XIII. and XIV.-Both females, married, born in Irelandone aged 40, the other 19 . Both afflicted with bronchoceles, which had commenced their growth in this country. Neither would submit to an operation.

CASES XV. and XVI.-Both of syphilitic sarcocele. These steadily improved, being treated externally by mercurial ointment, and internally by the syrup of the iodide of iron and iodide of potassium.

CASes XVII., XVIII. and XIX.-In all three, suppuration and abscess had resulted from boring the lobes of the cars.

\title{
ON SOME OF THE EFFECTS OF BROMIDE OF POTASSIUM.
}

\section{By Willinx Otis Jomnson, M.D.}

[Communicated for the Boston Medical and Surgicat Journal.]

Some time during the past summer, I casually stated to the Editors of this Journal that there had recently occurred, in several instances in my practice, some unusual symptoms immediately following the administration of medium doses of bromide of potassium. Up to that time, no similar obscrvation had found its way into print; but the November number of the Edinburgh Medical Journal mentions an instance of temporary paralysis, and alludes to analogous symptoms from this medicine, as reported in the Comptes Rendus and Centralblatc. Meantime, in October last, I observed a fourth case, and herewith report them all.

CASE I.-Female, single, æt. 59. Hysteria and asomnia. Had frequently taken bromide of potassium in moderato doses (eight to ten grains), with satisfactory results; had gono through with the eruption. Kept a solution on hand to use pro ve nata, herself the judgo as to occasion and quantity. Reported to me one morning that on the erening before, after two or three repetitions of the above dose (under thirty grains in all), she had felt a strange sensation of sinking, tremor, and anxicty, with numbness in legs and forcarms, lasting a few minutes and passing off gradually; no further effects, and no recurrence of these.

CASE II.-Male, æt. 28. Delirium tremons (epileptoid). All stimulus had been omitted for twelve hours, and the bromide substi. tuted, one drachm in solution, of which one fourth was ordered every hour; the four doses were given, and were succeeded by quict and repose of body and mind. At night, asomnia, restlessness and wan- 
dering returned. Thirty grains were given in one dose. Within five minutes there were increased agitation, tremors, anxicty, hysterical dyspnoa, numbness in limbs, loss of power in forearms, and a general subjective sense of immobility. Cessation of symptoms after a few minutes, and a comparatively quict night.

CaSE III.-Female, xt. 49. Widowed twelve years. Irritable and swollen os uteri from over-cauterization, with the whole list of reflex or peripheral spinal symptoms. Had been taking for some time fifteen grains of bromide of potassium at night, and more when necessary; effects always good. Took it regularly, and had the eruption. One night, quite late, I was sent for, to hear the report that after taking the bromide in somewhat larger dose than usual, between 10 and 11 o'clock, she had been seized with vertigo (to which she was normally subject, however), great agitation, trembling, numbness up and down the spinal column, and other indescribable sensations in back, loss of power in forearms, and a general apprehension of impending dissolution. None of these symptoms, however, awaited my arrival, and although they were reported the next morning as having continued during the night, the result of close questioning did not sustain her impression to that effect, and I was satisfied that they had neither continued nor recurred.

CaSe IV.-Male, æt. 40. Depression, asomnia, incertitude, irritability, insequence of thought. Had never taken bromide of potassium, nor used any narcotic or sedative. First dose at 8, P.M., fifteen grains; no result. Second at 9, thirty grains. A fow minutes afterwards, the patient sprang up in bed, apparently frightened, agitated, trembling; said that his legs were immovalle and his arms numb. A person in attendance fancied that a convulsion was at hand, and started for wine. Before it was ready, the symptoms had begun to subside; the stimulus was taken, however, and a quict night, without sleep, followed. The patient was afraid to try the bromide again, but there was no further occasion for it in the case.

Now to these facts, I add:-

1. That they are the only instances in an employment of this drug extending over a period of six years, and to not less than a hundred cases.

2. That the symptoms in other instances have not followed very much larger doses.

3. That they never returned after once subsiding; nor was the remedy suspended in consequence of them, nor did they ever repeat themselves in the same individual.

4. That the.phenomena reported attach to but a single point in observation, and are in too direct and apparently causative relation with that point to admit the suggestion of accident or coincidence.

5. And, finally, that they constitute, as here detailed, the only observation on this point as yet placed on permanent record. 